\title{
An Analysis for Survival Predictors for Patients with Hepatocellular Carcinoma Who Failed to Sorafenib Treatment in Pre-regorafenib Era
}

\author{
Chan Uk Lee*, Young-Sun Lee*, Ji Hoon Kim, Minjin Lee, Sehwa Kim, Young Kul Jung, Yeon Seok Seo, \\ Hyung Joon Yim, Jong Eun Yeon, Kwan Soo Byun \\ Department of Internal Medicine, Korea University College of Medicine, Seoul, Korea
}

Received Feb. 15, 2019 Revised Jun. 24, 2019 Accepted Jul. 12, 2019
Background/Aims: Sorafenib is the standard treatment for patients with advanced hepatocellular carcinoma (HCC). We aimed to investigate the prognosis predictors and the role of second-line cytotoxic systemic chemotherapy (CSC) in patients with advanced HCC after sorafenib discontinuation in the pre-regorafenib era.

Methods: From 2007 to 2015 in the pre-regorafenib era, the medical records of $166 \mathrm{HCC}$ patients, who had permanently discontinued sorafenib, were retrospectively reviewed. For further analysis of survival factors after sorafenib treatment failure, we compared the survival of patients who had maintained liver function after second-line treatment with the best supportive care (BSC) group and selective BSC (SBSC) group.

Results: After discontinuation of sorafenib, median overall survival (OS) was 2.8 (1.9-3.7) months. The OS in patients who discontinued sorafenib due to adverse effect, progression, and poor clinical condition were 5.5 (2.4-8.6), 5.5 (2.2-8.9), and 0.9 (0.5-1.3) months, respectively $(P<0.001)$. The independent predictive factors of survival after sorafenib failure were serum level of bilirubin and albumin, $\alpha$-fetoprotein, discontinuation cause, and second-line CSC. In comparison with survival between second-line CSC and BSC group, the CSC group showed better survival outcome compared to the BSC group (10.6 vs. 1.6 months, $P<0.001$ ) and SBSC group (10.6 vs. 4.2 months, $P=0.023)$.

Conclusions: The survival after sorafenib failure in patients who discontinued sorafenib due to progression and adverse effects was significantly better than in those who discontinued treatment due to clinical deterioration. In the pre-regorafenib era, patients who received second-line CSC showed better survival than those who received only supportive care after sorafenib failure. (J Liver Cancer 2019;19:117-127)

Keywords: Hepatocellular carcinoma; Sorafenib; Chemotherapy

\section{Corresponding author : Ji Hoon Kim}

Division of Gastroenterology and Hepatology, Department of Internal Medicine, Korea University Guro Hospital, Korea University College of Medicine, 148 Gurodong-ro, Guro-gu, Seoul 08308, Korea

Tel. +82-2-2626-3011, Fax. +82-2-2626-1038

E-mail; kjhhepar@naver.com

https://orcid.org/0000-0003-3924-0434

\section{INTRODUCTION}

Hepatocellular carcinoma (HCC) is the fifth most commonly occurring cancer, but the second most fatal form of cancer around the world. ${ }^{1}$ The high mortality rate of this disease is due to delayed diagnosis of HCC and there is a limited therapeutic modality for patients with advanced HCC. ${ }^{2,3}$ Sorafenib, which inhibits multiple kinases targeting Raf1, B-

*Chan Uk Lee and Young-Sun Lee equally contributed this work. 
Raf, vascular endothelial growth factor receptors, and plateletderived growth factor receptors, ${ }^{4,5}$ is the first proven therapeutic drug in patients with advanced HCC that accompanies portal vein invasion and distant metastasis. ${ }^{6,7}$ However, sorafenib treatment increased median overall survival by 2.8 and 2.3 months only as compared to the placebo in two multicenter phase III trials. ${ }^{8,9}$

Although there were significant benefits with sorafenib treatment with respect to survival and disease control rate, most of the disease controlled patients showed stable disease such that the tumor size showed neither sufficient shrinkage to qualify as partial response nor sufficient increase to qualify as progressive disease. ${ }^{8-10}$ Moreover, the overall incidence of sorafenib-related adverse events was very high, such as handfoot skin reaction, diarrhea, and fatigue. These adverse events led to dose reduction or discontinuation of sorafenib, resulting in a decrease of the anti-tumor effect of sorafenib. There have been several clinical trials for the treatment of advanced HCC patients with other target agents, such as sunitinib, ${ }^{11}$ linifanib, ${ }^{12}$ and brivanib. ${ }^{13,14}$ However, these drugs failed to show any clinical benefit in the prolongation of median survival. Further, the role of cytotoxic systemic chemotherapy (CSC) in patients with advanced HCC was unclear because there is neither any cytotoxic drug nor a combination of chemotherapy agents which have proven survival benefit in wellcontrolled, randomized trials. ${ }^{15}$ Fortunately, recently another systemic agent of lenvatinib has been approved as a first-line therapy after phase 3 trial that showed non-inferiority compared with sorafenib in advanced HCC patients without main portal vein involvement. ${ }^{16}$ However, there was no available second-line treatment after failure of lenvatinib.

Although regorafenib was first approved as a second-line therapy in $2017^{17}$ and several other agents showed promising results, ${ }^{18-20}$ in the pre-regorafenib era, the prognosis of patients with advanced HCC was very poor such that the median survival was 4.1 months after permanent discontinuation of sorafenib. ${ }^{21}$ This poor prognosis resulted from poor liver function, shortage of studies about post-sorafenib prognosis, and absence of approved second-line therapeutic options. Therefore, studies that investigate the predictor of survival and the role of second-line CSC for patients after sorafenib discontinuation are needed for widening the therapeutic strategy in HCC patients with sorafenib failure. In this study, we analyzed prognostic factors for these patients including second-line CSC in the pre-regorafenib era.

\section{METHODS}

\section{Patients and data collection}

From 2007 to 2015, we identified a total of 259 patients who were treated with sorafenib for advanced HCC. The exclusion criteria were 1) patients that were maintaining sorafenib at time of analysis, 2) patients that were lost to follow up during sorafenib treatment, and 3) patients that were prescribed sorafenib for 14 days or below. We retrospectively collected medical records of the patients including demographic data, laboratory data, the etiology of HCC, Eastern Cooperative Oncology Group (ECOG) performance status, tumor characteristics, and reason for discontinuation of sorafenib (tumor progression, adverse event, and clinical decompensation).

\section{Treatment}

After discontinuation of sorafenib, clinicians estimated the status of patients based on ECOG performance status, liver function, and tumor status. When general status was favorable (ECOG performance status 0 or 1 ) and liver function was preserved (child class A), these patients were candidates for second-line CSC. Depending on their consent, the patients received second-line CSC. The patients who were not treated with CSC received best supportive care.

The regimen of second-line CSC was composed of doxorubicin, cisplatin, and capecitabine. Doxorubicin $60 \mathrm{mg} / \mathrm{m}^{2}$ and cisplatin $60 \mathrm{mg} / \mathrm{m}^{2}$ were administrated on day 1 intravenously and oral capecitabine was prescribed for 21 days. Each cycle was repeated every 4 weeks. For nadir follow up, patients visited the clinic and checked complete blood count (CBC) and white blood cell differential counts from day 7 to 10. Patients who had received systemic chemotherapy took chest computed tomography (CT) and abdominal CT every 
2 cycles for assessment of treatment response. In the case of tumor progression, some patients received conservative management while other patients received third-line systemic chemotherapy that was comprised gemcitabine and oxaliplatin.

\section{Follow-up and outcome assessment}

Every patient visited at least once a month and was tested for $\mathrm{CBC}$, electrolyte, blood urea nitrogen, creatinine, aspartate aminotransferase, alanine transaminase, total bilirubin, prothrombin time (PT). $\alpha$-fetoprotein (AFP), and protein induced by vitamin $\mathrm{K}$ absence or antagonist-II was checked once every 1-3 months. In patients receiving second-line CSC, chest and abdominal CT was taken every 2 cycles to evaluate the treatment response. Tumor response was evaluated using the modified Response Evaluation Criteria in Solid Tumor (mRECIST) for HCC. ${ }^{22}$ According to mRECIST, tumor responses were categorized as complete response (CR), partial response (PR), stable disease (SD), and progressive disease $(\mathrm{PD})$. The overall response was determined as the number of patients with CR and PR out of total patients. Disease control rate was determined as the number of patients with CR, PR, and SD out of total patients. Adverse events were summarized according to the National Cancer Institute Common Terminology Criteria for Adverse Events version $5.0 .^{23}$

\section{Statistical analysis}

The baseline characteristics of patients were presented as mean or number of patients, as appropriate. Median overall survival (OS) period was calculated using the Kaplan-Meier curve and survival probability was compared by the log-rank test. A cox's regression model was used for evaluation of the significant factors affecting the prognosis after discontinuation of sorafenib. Multicollinearity analysis was performed and it was considered acceptable when variance inflation factor (VIF) values were less than 10 . All statistics were analyzed with SPSS ver. 24 (IBM Corp., Armonk, NY, USA). When a $P$-value was below 0.05 , it was considered statistically significant.

\section{RESULTS}

\section{Patients and baseline characteristics}

There were 259 patients who had been prescribed sorafenib due to advanced HCC (Fig. 1). Among them, 19 patients were maintaining sorafenib at the time of analysis, 60 patients were lost to follow-up during treatment with sorafenib, and 14 patients were prescribed sorafenib for 14 days or below. As a result, 166 patients were enrolled in this study. Among 89 patients who were a candidate for chemotherapy (Child-Pugh class A and ECOG 0-2), 41 patients were treated with systemic chemotherapy, four patients were treated with transcatheter arterial chemoembolization (TACE), and 44 patients received best supportive care as they refused further treatment. Among 77 patients who were not a candidate for chemotherapy, most patients received best supportive care (70 patients), but seven patients were treated with systemic chemotherapy because these patients insisted on further treatment. Finally, 114 patients received supportive care,

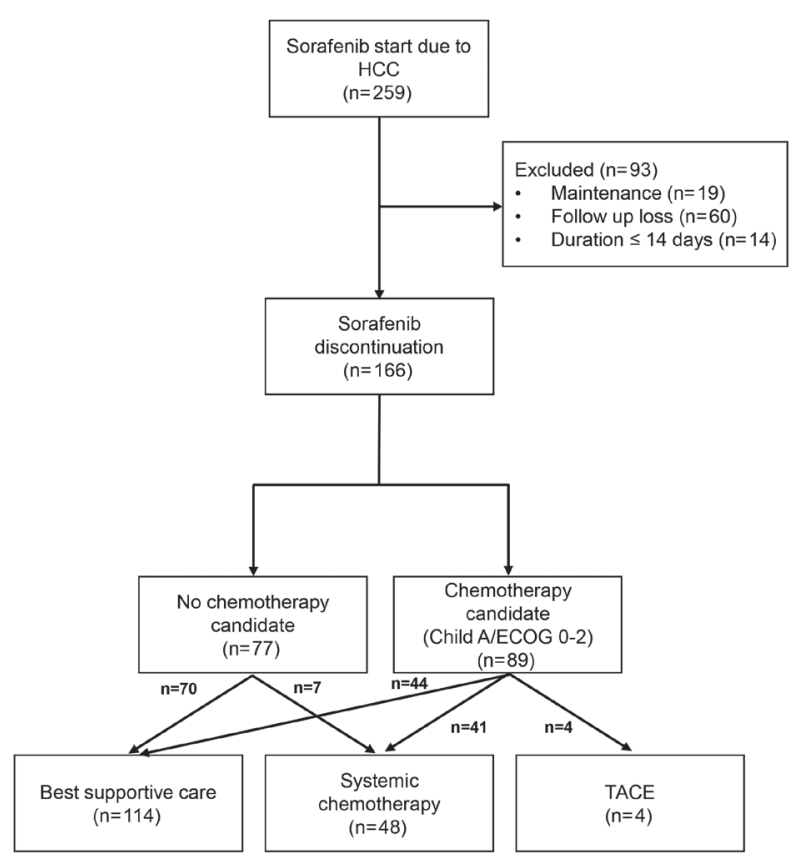

Figure 1. Flow diagram for study assessment. Initially, 1,635 reports were searched through the database and other sources. Only 25 studies were enrolled after evaluation. HCC, hepatocellular carcinoma; ECOG, Eastern Cooperative Oncology Group; TACE, transcatheter arterial chemoembolization. 
48 patients received second-line CSC, and four patients received TACE.

Baseline characteristics of 166 patients are summarized in Table 1. The mean age of the patients was 57.9 years and patients were predominantly men (81.9\%). Hepatitis B virus (HBV) was the main etiologic factor for the development of HCC (74.1\%), followed by alcohol consumption (12.7\%) and hepatitis C virus (HCV) (6.6\%). Most patients maintained good ECOG performance status $(0 / 1,63.2 \%)$ and only 54 patients $(32.5 \%)$ received sorafenib as an initial treat-

Table 1. Baseline characteristics

\begin{tabular}{|c|c|}
\hline Characteristic & Value $(n=166)$ \\
\hline Age (years) & $57.9 \pm 10.1$ \\
\hline Male & $136(81.9)$ \\
\hline \multicolumn{2}{|l|}{ Etiology } \\
\hline Alcohol & $21(12.7)$ \\
\hline HBV & $123(74.1)$ \\
\hline $\mathrm{HCV}$ & $11(6.6)$ \\
\hline Others & $11(6.6)$ \\
\hline \multicolumn{2}{|l|}{ ECOG PS } \\
\hline 0,1 & $105(63.2)$ \\
\hline $2,3,4$ & $61(36.8)$ \\
\hline SFN as initial therapy & $54(32.5)$ \\
\hline Ascites & $74(44.6)$ \\
\hline HEP & $13(7.8)$ \\
\hline Albumin (g/dL) & $3.5 \pm 0.6$ \\
\hline Total bilirubin (mg/dL) & $3.4 \pm 4.6$ \\
\hline PT (INR) & $1.3 \pm 0.4$ \\
\hline AFP $\geq 400 \mathrm{ng} / \mathrm{dL}$ & $95(57)$ \\
\hline PIVKA-II >200 mAU/mL & $138(83)$ \\
\hline Vascular invasion & $96(57.8)$ \\
\hline Distant metastasis & $135(81.3)$ \\
\hline \multicolumn{2}{|l|}{ Reason for discontinuation } \\
\hline Disease progression & $74(44.6)$ \\
\hline AE/self discontinuation & $31(18.7)$ \\
\hline Decompensation/poor condition & $61(36.7)$ \\
\hline
\end{tabular}

Values are presented as mean \pm standard deviation or number (\%). HBV, hepatitis B virus; HCV, hepatitis C virus; ECOG, Eastern Cooperative Oncology Group; PS, performance status; SFN, sorafenib; HEP, hepatic encephalopathy; PT, prothrombin time; INR, international normalized ratio; AFP, alpha fetoprotein; PIVKA-II, prothrombin in vitamin K absence-II; AE, adverse event. ment for HCC. Vascular invasion was observed in 96 patients (57.8\%) whereas 135 patients (81.3\%) had distant metastasis. Disease progression (44.6\%) and decompensation (36.7\%) were the main cause for discontinuation of sorafenib.

\section{Overall survival}

When we analyzed the OS of patients after discontinuation of sorafenib, the prognosis was very poor. The median OS was 2.8 month (95\% confidence interval [CI], 1.9-3.7) and

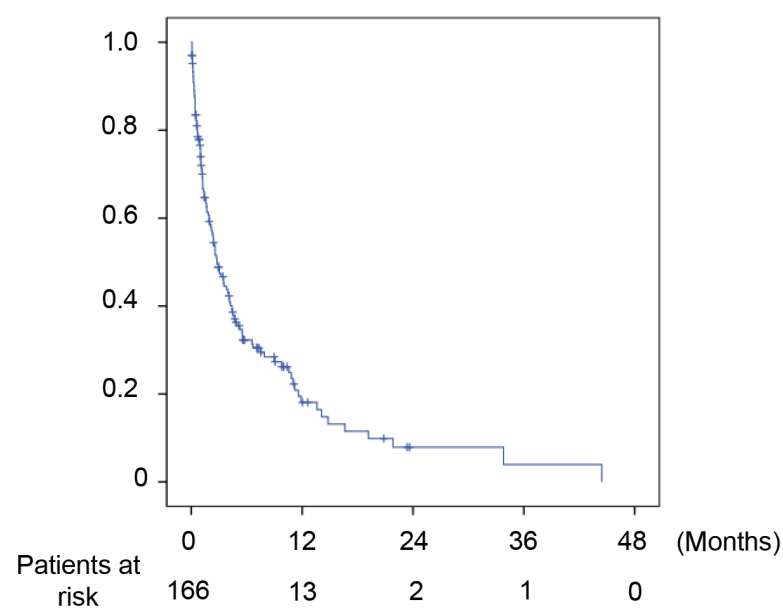

Figure 2. Kaplan-Meier curve of all patients that discontinue sorafenib permanently.

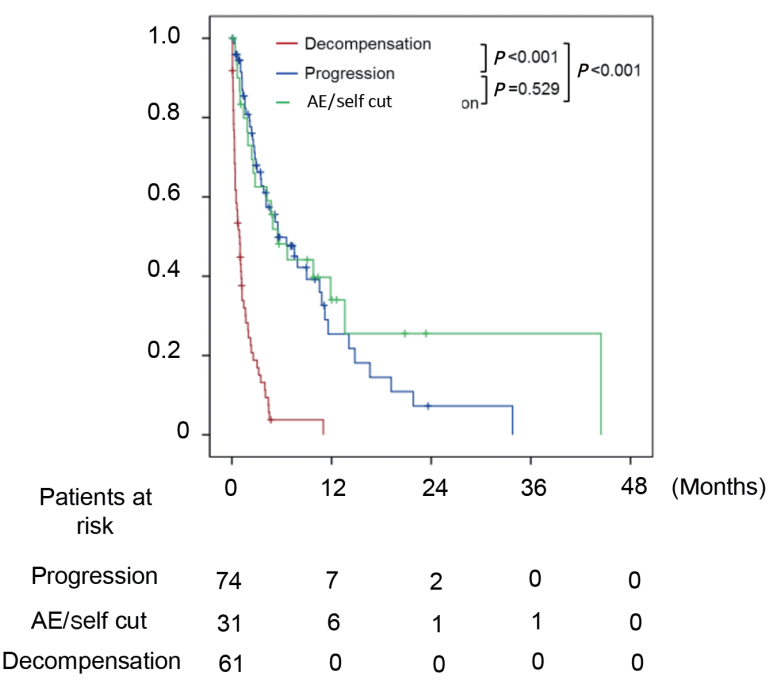

Figure 3. Kaplan-Meier curve of decompensation group (red), progression group (blue), and adverse event group (green). $A E$, adverse event. 
most of the patients (153 of 166 patients, 92.2\%) died within 12 months (Fig. 2). Next, we classified patients according to the reason for the discontinuation of sorafenib (Fig. 3). The median OS of decompensation group was 0.9 month $(95 \%$ $\mathrm{CI}, 0.5-1.3)$. The patients that discontinued sorafenib due to disease progression had a median OS of 5.6 months $(95 \%$ CI, 2.5-8.6). The median OS of the adverse event group was 5.5 months (95\% CI, 2.4-8.6). Compared to the decompensation group, the disease progression group $(P<0.001$ by logrank test) and adverse event group $(P<0.001$ by log-rank test) showed better OS. There was no significant difference between the disease progression group and adverse event group ( $P=0.529$ by log-rank test).

\section{Survival predictor of patients after discontinu- ation of sorafenib}

For analyzing survival predictor of patients after discontinuation of sorafenib, Cox regression analysis was performed (Table 2). In the univariate analysis, initial treatment, ECOG performance status, PT, bilirubin, albumin, AFP, ascites, hepatic encephalopathy, vascular invasion, the reason for discontinuation of sorafenib, and receiving of second-line treatment were significant. Because the reason for discontinuation of sorafenib and receiving of second-line treatment might influence each other, we conducted multivariate analysis thrice: first by model 1 which included the reason for discontinuation

Table 2. Univariate and multivariate analysis for survival factors after discontinuation of sorafenib

\begin{tabular}{|c|c|c|c|c|c|c|c|c|}
\hline Variable & $\begin{array}{l}\text { Univariate HR } \\
(95 \% \mathrm{Cl})\end{array}$ & $P$-value ${ }^{*}$ & $\begin{array}{l}\text { Multivariate HR } \\
(95 \% \mathrm{CI}) \text { model } 1\end{array}$ & $P$-value ${ }^{*}$ & $\begin{array}{l}\text { Multivariate HR } \\
(95 \% \mathrm{Cl}) \text { model } 2\end{array}$ & $P$-value ${ }^{*}$ & $\begin{array}{l}\text { Multivariate HR } \\
(95 \% \mathrm{Cl}) \text { model } 3\end{array}$ & $P$-value ${ }^{*}$ \\
\hline Sex (male) & $0.90(0.58-1.42)$ & 0.661 & - & - & - & - & & \\
\hline Initial Tx. (yes/no) & $1.73(1.18-2.53)$ & 0.005 & $0.87(0.57-1.33)$ & 0.523 & $1.22(0.80-1.86)$ & 0.353 & $1.220(0.791-1.882)$ & 0.368 \\
\hline $\begin{array}{l}\text { ECOG PS (2/3/4 vs. } \\
0 / 1)\end{array}$ & $4.83(3.25-7.18)$ & $<0.001$ & $1.02(0.45-2.33)$ & 0.964 & $1.679(0.96-2.94)$ & 0.071 & $0.941(0.417-2.125)$ & 0.884 \\
\hline PT & $5.18(3.46-7.74)$ & $<0.001$ & $1.45(0.75-2.82)$ & 0.268 & $1.45(0.75-2.80)$ & 0.276 & $1.494(0.777-2.783)$ & 0.229 \\
\hline Bilirubin & $1.20(1.15-1.24)$ & $<0.001$ & $1.16(1.10-1.22)$ & $<0.001$ & $1.15(1.09-1.21)$ & $<0.001$ & $1.153(1.095-1.215)$ & $<0.001$ \\
\hline Albumin & $0.37(0.29-0.48)$ & $<0.001$ & $0.58(0.41-0.84)$ & 0.004 & $0.59(0.40-0.85)$ & 0.005 & $0.624(0.427-0.913)$ & 0.015 \\
\hline $\begin{array}{l}\operatorname{AFP}(\geq 400 \text { vs. }<400 \\
n g / m L)\end{array}$ & $1.79(1.23-2.60)$ & 0.002 & $1.64(1.08-2.49)$ & 0.021 & $1.599(1.06-2.42)$ & 0.026 & $1.607(1.052-2.455)$ & 0.028 \\
\hline $\begin{array}{l}\text { PIVKA II ( } \geq 200 \text { vs. } \\
<200 \mathrm{mAU} / \mathrm{mL} \text { ) }\end{array}$ & $1.41(0.83-2.39)$ & 0.203 & - & - & - & - & & \\
\hline Ascites (yes/no) & $1.93(1.34-2.77)$ & $<0.001$ & $0.72(0.46-1.12)$ & 0.72 & $0.78(0.50-1.22)$ & 0.277 & $0.719(0.454-1.138)$ & 0.159 \\
\hline HEP (yes/no) & $2.77(1.48-5.20)$ & 0.002 & $0.99(0.49-1.99)$ & 0.984 & $0.96(0.48-1.91)$ & 0.905 & $0.999(0.499-2.000)$ & 0.998 \\
\hline $\begin{array}{l}\text { Vascular invasion } \\
\text { (yes/no) }\end{array}$ & $1.84(1.27-2.68)$ & 0.001 & $0.92(0.59-1.44)$ & 0.712 & $0.92(0.59-1.44)$ & 0.714 & 0.981 (0.624-1.541) & 0.934 \\
\hline Metastasis (yes/no) & $0.81(0.51-1.28)$ & 0.375 & - & - & - & - & & \\
\hline \multicolumn{9}{|l|}{$\begin{array}{l}\text { Reason for } \\
\text { discontinuation }\end{array}$} \\
\hline $\begin{array}{l}\text { Tumor } \\
\text { progression }\end{array}$ & - & - & - & - & - & - & & \\
\hline Adverse event & $0.84(0.9-1.44)$ & 0.529 & $0.89(0.52-1.55)$ & 0.686 & - & - & $0.831(0.470-1.470)$ & 0.525 \\
\hline $\begin{array}{l}\text { Clinical } \\
\text { decompensation }\end{array}$ & $4.95(3.22-7.59)$ & $<0.001$ & $2.34(1.023-5.32)$ & 0.043 & - & - & $1.997(0.878-4.541)$ & 0.099 \\
\hline $\begin{array}{l}\text { 2nd line treatment } \\
\text { (yes/no) }\end{array}$ & $0.28(0.18-0.43)$ & $<0.001$ & - & - & $0.57(0.33-0.98)$ & 0.040 & $0.543(0.302-0.974)$ & 0.040 \\
\hline
\end{tabular}

HR, hazard ratio; Cl, confidence interval; TX., therapy; ECOG, Eastern Cooperative Oncology Group; PS, performance status; PT, prothrombin time; AFP, alpha fetoprotein; PIVKA-II, prothrombin in vitamin K absence-II; HEP, hepatic encephalopathy.

"Cox's regression model was used. 
of sorafenib, second by model 2 which included second-line treatment, and third by model 3 which included both the reason for discontinuation and second-line treatment. In model 1, serum bilirubin level, serum albumin level, AFP, and clinical decompensation were significant prognostic factors for survival predictor of patients after discontinuation of sorafenib. In model 2, serum bilirubin level, serum albumin level, AFP, and second-line treatment were significant prognostic factors. In model 3, serum bilirubin, serum albumin, AFP level were significant prognostic factors. In multicollinearity analysis, VIF of the reason for discontinuation of sorafenib and receiving of second-line treatment were acceptable (1.360-1.643). There was no significant difference with respect to clinical decompensation, however, second-line treatment was still a significant prognostic factor for survival predictor of patients after discontinuation of sorafenib.

\section{Survival benefit of second-line systemic che- motherapy compared to best supportive care}

Next, we compared prognosis between the best supportive care group and second line systemic chemotherapy group after discontinuation of sorafenib. A total of 114 patients received best supportive care after discontinuation of sorafenib and median OS was 1.63 months (Fig. 4A). For the 48 patients who were treated with second-line systemic chemotherapy, the median OS was 10.57 months. Median OS showed a significant difference $(P<0.001$ by log-rank test).

However, the best supportive care group included 70 patients who had discontinued sorafenib due to liver decompensation and these patients could not receive second-line systemic chemotherapy. Therefore, we excluded these patients and compared the median OS between selective best supportive care group and second-line systemic chemotherapy group. Characteristics of the selective best supportive care group and chemotherapy group are summarized in Table 3. Chemotherapy group showed lower age, higher albumin level, and low total bilirubin level compared to selective best supportive care group. Second-line chemotherapy group also showed better OS comparing to selective supportive care group ( 10.57 months vs. 4.34 months, $P=0.023$ by log-rank test) (Fig. 4B).

\section{Clinical outcomes of advanced HCC patients who received second-line systemic chemo- therapy after discontinuation of sorafenib}

We analyzed the response rate of 48 HCC patients who re-
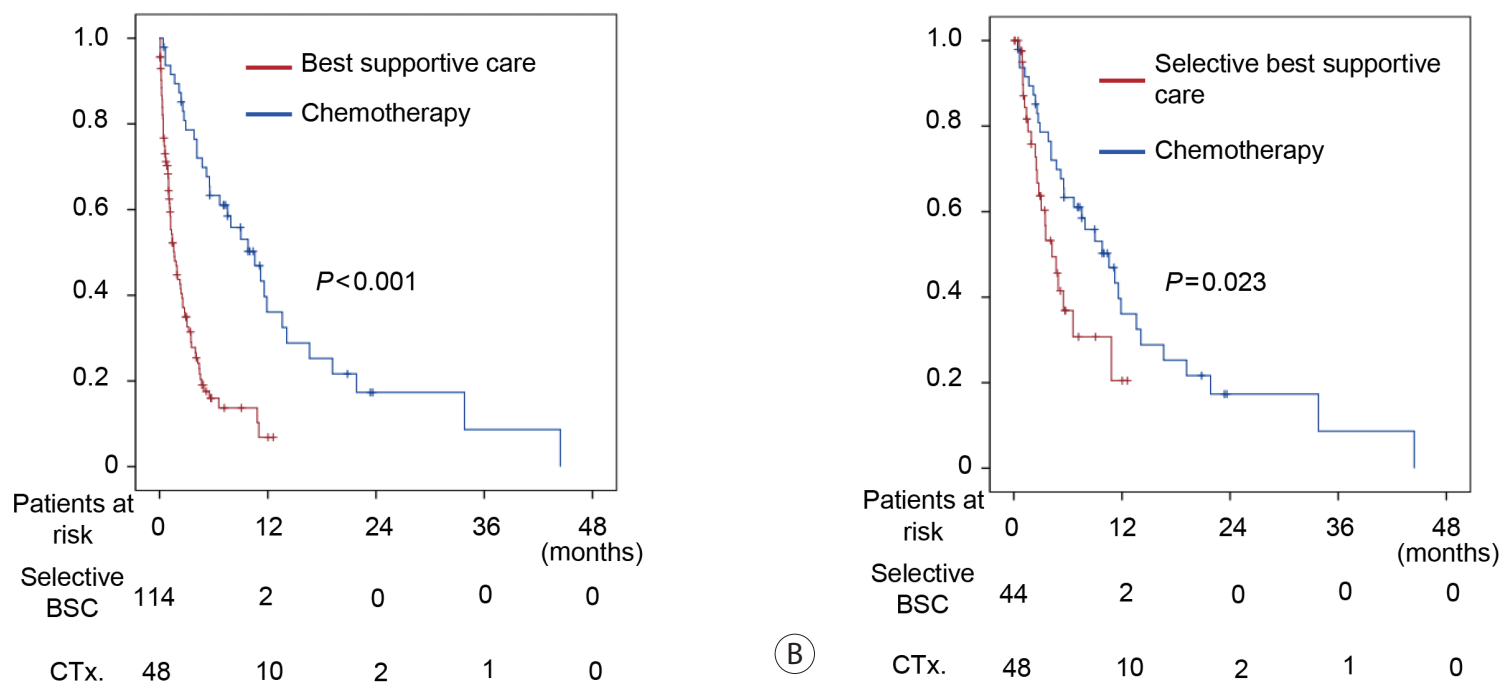

Figure 4. Kaplan-Meier analysis of overall survival (OS). (A) Median OS was 1.63 months in the best supportive care group (red) and 10.57 months in second-line chemotherapy group (blue). (B) Median OS was 4.34 months in the selective best supportive care group (red) and 10.57 months in second-line chemotherapy group (blue). BSC, best supportive care; CTx., chemotherapy. 
ceived second-line systemic chemotherapy after sorafenib failure (Table 4). When best response rates were analyzed excluding eight patients who were not available for tumor response, there was no CR, 10 patients (20.8\%) had PR, and 14 patients (29.2\%) had SD. However, 16 patients (33.3\%) had PD. The objective response rate was $20.8 \%$ and disease control rate was 50.5\%. Treatment-related adverse events are summarized in Table 5. In total, 47 patients (97.9\%) developed adverse events and 27 patients (56.3\%) developed grade $\geq 3$ adverse events. Treatment-related mortality was reported in five patients due to severe infection.
Table 4. Response rates after 2nd line chemotherapy

\begin{tabular}{lc}
\hline Response & Patient $(\mathbf{n}=\mathbf{4 8})$ \\
\hline Best response rates & \\
Complete response & $0(0.0)$ \\
Partial response & $10(20.8)$ \\
Stable disease & $14(29.2)$ \\
Disease progression & $16(33.3)$ \\
Not available & $8(16.7)$ \\
Objective response rates & $10(20.8)$ \\
Disease control rates & $24(50.5)$ \\
\hline
\end{tabular}

Values are presented as number (\%).

Table 3. Comparison between best supportive group and chemotherapy group

\begin{tabular}{|c|c|c|c|}
\hline Characteristic & BSC group $(n=44)$ & CTx. group $(n=48)$ & $P$-value ${ }^{*}$ \\
\hline Age (years) & $60.5 \pm 11.0$ & $55.4 \pm 9.8$ & 0.020 \\
\hline Male & $36(81.8)$ & 39 (81.3) & 0.944 \\
\hline \multicolumn{4}{|l|}{ Etiology } \\
\hline Alcohol & $7(15.9)$ & $6(12.5)$ & \\
\hline HBV & $29(65.9)$ & $37(77.1)$ & 0.695 \\
\hline $\mathrm{HCV}$ & $4(9.1)$ & $3(6.3)$ & \\
\hline Others & $4(9.1)$ & $2(4.2)$ & \\
\hline \multicolumn{4}{|l|}{ ECOG PS } \\
\hline 0,1 & $43(97.7)$ & $47(97.9)$ & 0.950 \\
\hline $2,3,4$ & $1(2.3)$ & $1(2.1)$ & \\
\hline SFN as initial therapy & $30(68.2)$ & $38(79.2)$ & 0.339 \\
\hline Ascites & $16(36.4)$ & $14(29.2)$ & 0.462 \\
\hline HEP & $1(2.3)$ & $0(0.0)$ & 0.294 \\
\hline Albumin (g/dL) & $3.6 \pm 0.4$ & $3.9 \pm 0.5$ & 0.002 \\
\hline Total bilirubin (mg/dL) & $1.8 \pm 1.6$ & $1.2 \pm 0.9$ & 0.010 \\
\hline PT (INR) & $1.14 \pm 1.18$ & $1.11 \pm 0.16$ & 0.365 \\
\hline AFP $\geq 400 \mathrm{ng} / \mathrm{dL}$ & $25(56.8)$ & $22(45.8)$ & 0.292 \\
\hline PIVKA-II >200 mAU/mL & $32(76.2)$ & 39 (81.3) & 0.557 \\
\hline Vascular invasion & $23(52.3)$ & $22(45.8)$ & 0.537 \\
\hline Distant metastasis & $38(86.4)$ & $42(87.5)$ & 0.872 \\
\hline \multicolumn{4}{|l|}{ Reason for discontinuation } \\
\hline Disease progression & $27(61.4)$ & $36(75.0)$ & 0.366 \\
\hline AE/self discontinuation & 17 (38.6) & $12(25.0)$ & \\
\hline
\end{tabular}

Values are presented as mean \pm standard deviation or number (\%).

BSC, best supportive care; CTx., chemotherapy; HBV, hepatitis B virus; HCV, hepatitis C virus; ECOG, Eastern Cooperative Oncology Group; PS, performance status; SFN, sorafenib; HEP, hepatic encephalopathy; PT, prothrombin time; INR, international normalized ratio; AFP, alpha fetoprotein; PIVKA-II, prothrombin in vitamin Kabsence-II; AE, adverse event.

*The Chi-square test and Fisher's extract test were used. 
Table 5. Summary of chemotherapy-related adverse events

\begin{tabular}{lcc}
\hline Adverse event & $\begin{array}{c}\text { Any grade } \\
(\mathbf{n}=\mathbf{4 8})\end{array}$ & $\begin{array}{c}\text { Grade } \geq \mathbf{3} \\
(\mathbf{n}=\mathbf{4 8})\end{array}$ \\
\hline Overall incidence & $47(97.9)$ & $27(56.3)$ \\
\hline Anorexia & $18(37.5)$ & $0(0.0)$ \\
\hline Nausea & $23(47.9)$ & $0(0.0)$ \\
Vomiting & $10(20.3)$ & $0(0.0)$ \\
\hline Diarrhea & $7(14.6)$ & $0(0.0)$ \\
\hline Abdominal pain & $27(56.3)$ & $0(0.0)$ \\
\hline Abdominal distension & $5(10.4)$ & $0(0.0)$ \\
\hline Stomatitis & $16(33.3)$ & $0(0.0)$ \\
\hline Gl bleeding & $3(6.3)$ & $3(6.3)$ \\
\hline Anemia & $33(68.8)$ & $16(33.3)$ \\
\hline Neutropenic fever & $20(41.7)$ & $20(41.7)$ \\
\hline Transaminase elevation & $7(14.6)$ & $3(6.3)$ \\
\hline Hyperbilirubinemia & $6(12.5)$ & $2(4.2)$ \\
\hline Infection & $13(27.1)$ & $13(27.1)$ \\
\hline Treatment-related mortality & $5(10.4)$ & $5(10.4)$ \\
\hline
\end{tabular}

Values are presented as number (\%).

\section{DISCUSSION}

Although the survival benefit of sorafenib has been shown by various studies, ${ }^{8,9}$ the prognosis of patients who discontinue sorafenib permanently was very poor. Iavarone et al. ${ }^{21}$ reported that discontinuation of sorafenib due to adverse events, absence of macrovascular invasion, extrahepatic metastasis, and poor performance status were predictors of survival after discontinuation of sorafenib. After discontinuation of sorafenib, some patients were capable of receiving second-line treatment, especially those who discontinued sorafenib due to progressive disease or severe adverse events. For justification of second-line treatment, studies that evaluate the survival predictor are needed including whether patients received second-line treatment.

In the treatment of HCC, clinicians should consider several factors including tumor stage, performance status, and especially, liver function. According to recent modified Barocelona Clinic Liver Cancer BCLC algorithm, systemic treatment was recommended in the advanced stage that represents portal vein invasion and extrahepatic metastasis with performance status 1 or 2 and Child-Pugh class A. ${ }^{6}$ In case of discontinuation of sorafenib, Iavarone et al. ${ }^{21}$ reported that $23 \%$ of the patients discontinued sorafenib due to liver decompensation. Likewise, $36.7 \%$ of the patients discontinued sorafenib due to liver decompensation or poor ECOG performance status in our population and these patients showed poor prognosis. Liver decompensation or poor general condition could be a critical factor in patients with HCC. However, discontinuation of sorafenib due to tumor progression or adverse events were good survival predictors and these patients could be ideal candidates for second-line treatment. ${ }^{24}$ Further, our results showed that adverse events and tumor progression were good prognostic factors compared to clinical decompensation. In addition, serum bilirubin level, serum albumin level, and AFP were also significant factors influencing survival. In HCC patients, laboratory tests were important because some of them represent liver function. Serum levels of bilirubin and albumin are markers that represent liver function, thus these might act as predictors of survival. AFP is a tumor marker for HCC surveillance and is associated with prognosis when present at high levels. ${ }^{25}$ Likewise, the elevation of AFP level (above $400 \mathrm{ng} / \mathrm{dL}$ ) is a significant prognostic factor in this study.

For the development of second-line treatment after sorafenib discontinuation in advanced HCC patients, other agents were investigated including brivanib, ${ }^{14}$ everolimus, ${ }^{26}$ and ramucirumab. ${ }^{27}$ Unfortunately, these agents failed to improve OS and increased adverse events. Although Iavarone et al. ${ }^{21}$ reported that median post-sorafenib survival (PSS) was only 1.8 months in patients with worsening liver function, PSS of the adverse event group was 7.3 months and that of tumor progression group was 4.6 months. Therefore, second-line therapy could be provided to these groups. In recent phase 3 trials, regorafenib, which is an oral multi-kinase inhibitor targeting various kinases involved in angiogenesis and tumorigenesis, ${ }^{28}$ showed acceptable tolerability and significant survival benefit in patients with advanced HCC progressing on sorafenib as compared to the placebo. ${ }^{17}$ Regorafenib increased OS in patients with metastatic colorectal cancer $^{29}$ and improved progression-free survival in patients with advanced gastrointestinal stromal tumor. ${ }^{30}$ However, several adverse events have been reported including hand- 
foot skin reaction, hypertension, fatigue, and diarrhea. ${ }^{31}$ These adverse events are similar to those observed in the case of sorafenib since they share a similar mechanism. As a result, the sorafenib discontinuation patients would experience the same adverse events. Recent phase III trials included only those HCC patients who had radiologic progression excluding sorafenib intolerable patients. In addition, the high cost would be a major obstacle in the usage of regorafenib in patients with advanced HCC. Therefore, other second-line therapeutic modalities are needed other than regorafenib, especially for patients that discontinue sorafenib due to adverse events.

Although systemic chemotherapy has been tried for advanced HCC patients, its role is still unclear. ${ }^{15}$ Doxorubicinbased therapy, ${ }^{32,33}$ cisplatin-based therapy, ${ }^{34}$ and FOLFOX regimen ${ }^{35}$ showed a beneficial effect for advanced HCC patients. In 3 phase RCT comparison between nolatrexed and doxorubicin, doxorubicin showed significantly higher OS (34.3 weeks) than the nolatrexed group (22.3 weeks). ${ }^{33} \mathrm{Al}$ though combination therapy with cisplatin, interferon $\alpha-2 b$, doxorubicin, and fluorouracil treatment showed increased survival compared with doxorubicin monotherapy, this was not significant. ${ }^{34}$ However, most cytotoxic chemotherapies showed limited prolongation of median OS and there is no satisfactory randomized controlled trial. Moreover, recent advancement in the development of target agent constricts investigation for cytotoxic chemotherapy. Our result showed that doxorubicin-based chemotherapy increased median OS compared to control and represented a satisfactory objective response rate (20.8\%) and disease control rate (50.5\%). Therefore, CSC could be a therapeutic option after sorafenib discontinuation in patients with advanced HCC in the preregorafenib era.

Because our study is retrospective, chemotherapy group and supportive care group were not randomized. Therefore, there is a possibility of selection bias because the decision for second-line chemotherapy was determined individually. Propensity scoring match would be the best option between the best supportive care group and second-line chemotherapy group, but it was impossible due to the small number of patients in this study. To compensate for the selection bias, we classified the selective supportive care group that had favorable performance status and preserved liver function and compared survival between second-line chemotherapy group and selective supportive care group. Moreover, because recent second-line therapy has been approved and is in development, the implication of this study should be interpreted as restricted survival analysis of sorafenib failure limited to the pre-regorafenib era.

In conclusion, serum bilirubin level, serum albumin level, AFP, the reason for discontinuation of sorafenib, and receiving of second-line treatment were significant survival predictors in patients who discontinued sorafenib permanently. The survival of patients who discontinue sorafenib due to progression and adverse effects was significantly better than those who discontinued due to clinical deterioration. Moreover, patients who received second-line cytotoxic chemotherapy showed better survival than those who received only supportive care. Therefore, second-line CSC might be considered in patients who discontinue sorafenib permanently. However, recently developed second-line therapies of regorafenib, ${ }^{17}$ cabozantinib, ${ }^{18}$ ramucirumab, ${ }^{20}$ and nivolumab ${ }^{19}$ should be compared with existing second-line cytotoxic therapies to determine clinical feasibility.

\section{ACKNOWLEDGEMENT}

This work was supported by an academic research fund of the Korean Liver Cancer Study for 2016 and the National Research Foundation of Korea (NRF) grant funded by the Korea government (the Ministry of Education, Science and Technology) (2015R1C1A1A01052360).

\section{Conflicts of Interest}

The authors have no conflicts to disclose.

\section{REFERENCES}

1. Ferlay J, Soerjomataram I, Dikshit R, Eser S, Mathers C, Rebelo $M$, et al. Cancer incidence and mortality worldwide: sources, methods and major patterns in GLOBOCAN 2012. Int J Cancer 2015;136:E359-386. 
2. Llovet JM, Brú C, Bruix J. Prognosis of hepatocellular carcinoma: the BCLC staging classification. Semin Liver Dis 1999;19:329-338.

3. Forner $A$, Reig ME, de Lope CR, Bruix J. Current strategy for staging and treatment: the BCLC update and future prospects. Semin Liver Dis 2010;30:61-74.

4. Wilhelm SM, Carter C, Tang L, Wilkie D, McNabola A, Rong H, et al. BAY 43-9006 exhibits broad spectrum oral antitumor activity and targets the RAF/MEK/ERK pathway and receptor tyrosine kinases involved in tumor progression and angiogenesis. Cancer Res 2004;64:7099-7109.

5. Wilhelm SM, Adnane L, Newell P, Villanueva A, Llovet JM, Lynch $M$. Preclinical overview of sorafenib, a multikinase inhibitor that targets both Raf and VEGF and PDGF receptor tyrosine kinase signaling. Mol Cancer Ther 2008;7:3129-3140.

6. European Association for the Study of the Liver. EASL Clinical practice guidelines: management of hepatocellular carcinoma. J Hepatol 2018;69:182-236.

7. Marrero JA, Kulik LM, Sirlin CB, Zhu AX, Finn RS, Abecassis MM, et al. Diagnosis, staging, and management of hepatocellular carcinoma: 2018 practice guidance by the American Association for the Study of Liver Diseases. Hepatology 2018;68:723-750.

8. Llovet JM, Ricci S, Mazzaferro V, Hilgard P, Gane E, Blanc JF, et al. Sorafenib in advanced hepatocellular carcinoma. N Engl J Med 2008;359:378-390.

9. Cheng AL, Kang YK, Chen Z, Tsao CJ, Qin S, Kim JS, et al. Efficacy and safety of sorafenib in patients in the Asia-Pacific region with advanced hepatocellular carcinoma: a phase III randomised, double-blind, placebo-controlled trial. Lancet Oncol 2009;10:25-34.

10. Eisenhauer EA, Therasse P, Bogaerts J, Schwartz LH, Sargent D, Ford $R$, et al. New response evaluation criteria in solid tumours: revised RECIST guideline (version 1.1). Eur J Cancer 2009;45:228247.

11. Cheng AL, Kang YK, Lin DY, Park JW, Kudo M, Qin S, et al. Sunitinib versus sorafenib in advanced hepatocellular cancer: results of a randomized phase III trial. J Clin Oncol 2013;31:4067-4075.

12. Cainap C, Qin S, Huang WT, Chung IJ, Pan H, Cheng Y, et al. Linifanib versus Sorafenib in patients with advanced hepatocellular carcinoma: results of a randomized phase III trial. J Clin Oncol 2015;33:172-179.

13. Johnson PJ, Qin SK, Park JW, Poon RTP, Raoul JL, Philip PA, et al. Brivanib versus sorafenib as first-line therapy in patients with unresectable, advanced hepatocellular carcinoma: results from the randomized phase III BRISK-FL Study. J Clin Oncol 2013;31:35173524.

14. Llovet JM, Decaens T, Raoul JL, Boucher E, Kudo M, Chang C, et al. Brivanib in patients with advanced hepatocellular carcinoma who were intolerant to sorafenib or for whom sorafenib failed: results from the randomized phase III BRISK-PS study. J Clin Oncol
2013;31:3509-3516.

15. Kim BH, Park JW. Recent advances in systemic chemotherapy of hepatocellular carcinoma. J Korean Med Assoc 2013;56:993-1000.

16. Kudo M, Finn RS, Qin S, Han KH, Ikeda K, Piscaglia F, et al. Lenvatinib versus sorafenib in first-line treatment of patients with unresectable hepatocellular carcinoma: a randomised phase 3 noninferiority trial. Lancet 2018;391:1163-1173.

17. Bruix J, Qin S, Merle P, Granito A, Huang YH, Bodoky G, et al. Regorafenib for patients with hepatocellular carcinoma who progressed on sorafenib treatment (RESORCE): a randomised, doubleblind, placebo-controlled, phase 3 trial. Lancet 2017;389:56-66.

18. Abou-Alfa GK, Meyer T, Cheng AL, El-Khoueiry AB, Rimassa $L$, Ryoo BY, et al. Cabozantinib in patients with advanced and progressing hepatocellular carcinoma. N Engl J Med 2018;379:54-63.

19. El-Khoueiry AB, Sangro B, Yau T, Crocenzi TS, Kudo M, Hsu C, et al. Nivolumab in patients with advanced hepatocellular carcinoma (CheckMate 040): an open-label, non-comparative, phase 1/2 dose escalation and expansion trial. Lancet 2017;389:2492-2502.

20. Zhu AX, Kang YK, Yen CJ, Finn RS, Galle PR, Llovet JM, et al. Ramucirumab after sorafenib in patients with advanced hepatocellular carcinoma and increased alpha-fetoprotein concentrations (REACH-2): a randomised, double-blind, placebo-controlled, phase 3 trial. Lancet Oncol 2019;20:282-296.

21. lavarone M, Cabibbo G, Biolato M, Della Corte C, Maida M, Barbara $M$, et al. Predictors of survival in patients with advanced hepatocellular carcinoma who permanently discontinued sorafenib. Hepatology 2015;62:784-791.

22. Lencioni R, Llovet JM. Modified RECIST (mRECIST) assessment for hepatocellular carcinoma. Semin Liver Dis 2010;30:52-60.

23. Yang K, Sung PS, Oh JS, Chun HJ, Jang JW, Bae SH, et al. Transarterial chemolipiodolization for hepatocellular carcinoma with central bile duct invasion causing conjugated hyperbilirubinemia: safety and prognostic factors for survival. J Liver Cancer 2018;18:121129.

24. Reig M, Rimola J, Torres F, Darnell A, Rodriguez-Lope C, Forner A, et al. Postprogression survival of patients with advanced hepatocellular carcinoma: rationale for second-line trial design. Hepatology 2013;58:2023-2031.

25. Farinati F, Marino D, De Giorgio M, Baldan A, Cantarini M, Cursaro $C$, et al. Diagnostic and prognostic role of alpha-fetoprotein in hepatocellular carcinoma: both or neither? Am J Gastroenterol 2006;101:524-532.

26. Zhu AX, Kudo M, Assenat E, Cattan S, Kang YK, Lim HY, et al. Effect of everolimus on survival in advanced hepatocellular carcinoma after failure of sorafenib: the EVOLVE-1 randomized clinical trial. JAMA 2014;312:57-67.

27. Zhu AX, Park JO, Ryoo BY, Yen CJ, Poon R, Pastorelli D, et al. Ramucirumab versus placebo as second-line treatment in patients 
with advanced hepatocellular carcinoma following first-line therapy with sorafenib (REACH): a randomised, double-blind, multicentre, phase 3 trial. Lancet Oncol 2015;16:859-870.

28. Wilhelm SM, Dumas J, Adnane L, Lynch M, Carter CA, Schütz G, et al. Regorafenib (BAY 73-4506): a new oral multikinase inhibitor of angiogenic, stromal and oncogenic receptor tyrosine kinases with potent preclinical antitumor activity. Int J Cancer 2011;129:245255.

29. Grothey A, Van Cutsem E, Sobrero A, Siena S, Falcone A, Ychou M, et al. Regorafenib monotherapy for previously treated metastatic colorectal cancer (CORRECT): an international, multicentre, randomised, placebo-controlled, phase 3 trial. Lancet 2013;381:303312.

30. Demetri GD, Reichardt P, Kang YK, Blay JY, Rutkowski P, Gelderblom $H$, et al. Efficacy and safety of regorafenib for advanced gastrointestinal stromal tumours after failure of imatinib and sunitinib (GRID): an international, multicentre, randomised, placebocontrolled, phase 3 trial. Lancet 2013;381:295-302.

31. De Wit M, Boers-Doets $C B$, Saettini $A$, Vermeersch $K$, de Juan $C R$, Ouwerkerk J, et al. Prevention and management of adverse events related to regorafenib. Support Care Cancer 2014;22:837-846.

32. Gish RG, Porta C, Lazar L, Ruff P, Feld R, Croitoru A, et al. Phase III randomized controlled trial comparing the survival of patients with unresectable hepatocellular carcinoma treated with nolatrexed or doxorubicin. J Clin Oncol 2007;25:3069-3075.

33. Yoon EL, Yeon JE, Lee HJ, Suh SJ, Lee SJ, Kang SH, et al. Systemic cytotoxic chemotherapy of patients with advanced hepatocellular carcinoma in the era of sorafenib nonavailability. J Clin Gastroenterol 2014;48:e22-e29.

34. Yeo W, Mok TS, Zee B, Leung TW, Lai PB, Lau WY, et al. A randomized phase III study of doxorubicin versus cisplatin/interferon alpha-2b/doxorubicin/fluorouracil (PIAF) combination chemotherapy for unresectable hepatocellular carcinoma. J Natl Cancer Inst 2005;97:1532-1538.

35. Qin S, Bai Y, Lim HY, Thongprasert S, Chao Y, Fan J, et al. Randomized, multicenter, open-label study of oxaliplatin plus fluorouracil/leucovorin versus doxorubicin as palliative chemotherapy in patients with advanced hepatocellular carcinoma from Asia. J Clin Oncol 2013;31:3501-3508. 